\title{
Correlação entre medidas de força e velocidade em jovens futebolistas
}

Correlation between strength and speed measures in young football players

Correlación entre medidas de fuerza y velocidad en jóvenes jugadores de futbol

\author{
Ezequias Pereira Neto ${ }^{1,4}$ \\ Jarlisson Francsuel Melo dos Santos ${ }^{1}$ \\ Leandro Henrique Brandão Albuquerque ${ }^{1}$ \\ Vanessa Marques Schmitzhaus ${ }^{1}$ \\ Antônio Bruno Magalhães Silva ${ }^{2}$ \\ Marzo Edir Da Silva-Grigoletto ${ }^{1}$ \\ Ragami Chaves Alves ${ }^{3}$ \\ Marcos Bezerra de Almeida ${ }^{1}$
}

\section{Resumo}

Introdução: Recentes estudos demonstram uma relação entre força máxima e desempenho de Sprint em atletas adultos; contudo, essa relação em atletas mais jovens foi menos investigada. Objetivo: Verificar a correlação entre o teste de força máxima, analisado pelo 1RM e a velocidade máxima linear, analisada pelo teste de Sprint de 30m. Metodologia: Dezoito jovens futebolistas $(18,1 \pm 1,3$ anos) realizaram medidas de força máxima (Teste de 1RM) e velocidade máxima linear (Sprint de $30 \mathrm{~m}$ ) em período pré competitivo. Para analisar as correlações entre as variáveis, foi utilizado a correlação de Spearman. Complementarmente foi realizado uma análise de inferência qualitativa. Resultados: Os resultados apontam relação inversa fraca entre força e velocidade $(r=-0,367)$ contudo, uma inferência qualitativa aponta que possivelmente haja essa relação inversa. Conclusão: Conclui-se que há uma relação fraca entre força e velocidade em jovens futebolistas.

Palavras - chave: Desempenho Atlético. Aceleração. Cineantropometria.

\section{Abstract}

Introduction: Recent studies demonstrate a relationship between maximum strength and Sprint performance in adult athletes; however, this relationship in younger athletes has been less investigated. Objective: To verify the correlation between the maximum strength test, analyzed by the 1RM and the maximum linear speed, analyzed by the $30 \mathrm{~m}$ Sprint test. Methodology: Eighteen young soccer players $(18.1 \pm 1.3$ years $)$ performed measurements of maximum strength (1RM test) and maximum linear speed (30m sprint) in a precompetitive period. To analyze the correlations between the variables, Spearman's correlation was used. In addition, a qualitative inference analysis was carried out. Results: The results show a weak inverse relationship between strength and speed $(r=-0.367)$, however, a qualitative inference points out that there is possibly an inverse relationship. Conclusion: It is concluded that there is a weak relationship between strength and speed in young soccer players.

Keywords: Athletic Performance. Acceleration. Cineanthropometry.

\section{Resumen}

Introducción: Estudios recientes demuestran una relación entre la fuerza máxima y el rendimiento de Sprint en atletas adultos; sin embargo, esta relación en atletas más jóvenes 
ha sido menos investigada. Objetivo: verificar la correlación entre la prueba de fuerza máxima, analizada por el 1RM y la velocidad lineal máxima, analizada por la prueba Sprint de $30 \mathrm{~m}$. Metodología: Dieciocho jóvenes futbolistas $(18.1 \pm 1.3$ años $)$ realizaron mediciones de fuerza máxima (prueba de 1RM) y velocidad lineal máxima (sprint de 30 $\mathrm{m}$ ) en un período precompetitivo. Para analizar las correlaciones entre las variables, se utilizó la correlación de Spearman. Además, se realizó un análisis de inferencia cualitativa. Resultados: Los resultados muestran una relación inversa débil entre fuerza y velocidad ( $\mathrm{r}$ $=-0.367)$, sin embargo, una inferencia cualitativa señala que posiblemente existe una relación inversa. Conclusión: se concluye que existe una relación débil entre la fuerza y la velocidad en los futbolistas jóvenes.

Palabras - clave: Rendimiento atlético. Aceleración Cineantropometría.

\section{INTRODUÇÃO}

O futebol é caracterizado como um esporte intermitente de alta intensidade, e essa característica aponta para priorizar taxas ótimas de desenvolvimento da força e da velocidade do atleta, estas, capacidades fundamentais para o sucesso no esporte ${ }^{1,2}$.

Diretrizes recentes admitem que programas de treinamento de força $(\mathrm{TF})$ sejam fundamentais para melhoria das capacidades físicas como a força e velocidade $^{3,4}$, assim favorecendo a evolução no desempenho do jovem atleta. No entanto, os ganhos mais expressivos dessas capacidades físicas, exigem modelos de treinamento cujo adoção de altas cargas, mesmo em indivíduos jovens, são fundamentais ${ }^{5}$.

As cargas de trabalho planejadas, exigem do treinador um controle de quantificação e monitoramento desta variável do $\mathrm{TF}^{6}$. De fato, a intensidade do TF torna-se uma variável fundamental para potencializar os ganhos neuromusculares do atleta ${ }^{7}$ Comumente, a utilização do teste de uma repetição máxima (1RM) é a mais adotada entre treinadores para mensurar a força máxima em programas de $\mathbf{T F}^{8}$.

Para medir o 1RM, o atleta necessita realizar um levantamento máximo com uma carga que possa ser movida uma única vez, caracterizando assim um teste de esforço extremo e sujeito a aplicabilidade prática discutível, logo, novas estratégias surgem para estimar indiretamente o 1RM de uma maneira menos exigente ${ }^{9,10}$. Medidas de velocidade de deslocamento da barra em diversos exercícios vem sendo apresentada como estimativas válidas para estimar o $1 \mathrm{RM}$ de um atleta, sem a realização do teste de $1 \mathrm{RM}^{10,11}$. Em adendo, é importante frisar que o desenvolvimento da curva forçavelocidade é de utilidade prática para propor novas estratégias de TF para atletas $^{12}$.

Em estudo conduzido por Weyand et al. $^{13}$, foi apontado uma correlação positiva entre força máxima de 
reação do solo e velocidade máxima de corrida $(r=0,600)$ em corredores, com isso os autores sugerem que a produção de força de máxima é parte integrante da produção de velocidade máxima linear. Esta relação entre força e velocidade, justifica uma plausível investigação entre os testes de 1RM e sua possível relação com a velocidade máxima linear.

Posto isso, hipotetizamos que possa haver uma relação entre as capacidades físicas de força máxima (medida pelo teste de 1RM) e velocidade máxima linear (medida pelo teste de Sprint de $30 \mathrm{~m}$ ) em jovens jogadores de futebol. Logo, sugere-se mensurar as taxas de desenvolvimento da velocidade ao longo da temporada, como uma forma menos exaustiva e mais ecológica para relacionar também aos ganhos de força.

O presente estudo tem como objetivo verificar a correlação entre o teste de força máxima, analisado pelo 1RM e a velocidade máxima linear, analisada pelo teste de Sprint de $30 \mathrm{~m}$.

\section{MATERIAIS E MÉTODOS}

Este estudo tem um desenho correlacional transversal para descrever e explorar as relações entre velocidade máxima linear (Sprint $30 \mathrm{~m}$ ) e força máxima (1RM). Todos os atletas estavam familiarizados com os procedimentos de teste, que eram realizados durante o período pré competitivo. Antes dos testes (executados em dias distintos) os atletas realizaram aquecimento geral e específico, respeitando o protocolo de cada teste. O projeto de pesquisa foi aprovado pelo Comitê de Ética da Universidade Federal de Sergipe, parecer n.? 3.493.367 e seguiu todas as normas de estudos com seres humanos, conforme as recomendações do CNS 96/96 da Associação Médica Mundial e a Declaração de Helsinki ${ }^{14}$.

Fizeram parte da amostra 18 atletas de futebol da categoria sub 20 (18,1 $\pm 1,3$ anos, IMC 21,9 $\pm 1,4$ kg.m-2). A rotina desses atletas era constituída de, aproximadamente, 1 hora diária de treinamento, cinco vezes por semana. A programação semanal durante a prétemporada era organizada em duas sessões de treino técnico-tático (60 min), e três sessões de treino físico (20 a 40 min). Foram convidados a participar do estudo atletas de futebol jovens com idade máxima de 20 anos (nascidos entre 1999 e 2003), vinculados a uma equipe registrada na Federação de Futebol Estadual local, mas que não tivessem participado de programa de treinamento de força nos seis meses anteriores à coleta. Seriam excluídos da amostra atletas que apresentassem fatores de risco cardiovascular ou lesões musculoesqueléticas que pudessem 
impedir ou limitar a realização dos procedimentos (nenhuma ocorrência).

O teste de 1RM foi desempenhado conforme descrito pelo National Strength and Conditioning Association', inclusive reproduzindo as estratégias sugeridas para minimizar margem de erro na execução. Desta forma, as instruções devem ser transmitidas a todos os atletas de forma padronizada, indicando cuidadosamente a técnica do movimento. Os pesos utilizados no estudo devem ser previamente aferidos em balança de precisão. Ao longo do teste, o avaliador deve permanecer atento durante toda a execução do movimento para evitar interpretações errôneas dos escores obtidos, e manter encorajamento verbal para que os participantes possam obter seu melhor desempenho. No presente estudo, o exercício agachamento foi utilizado para a determinação da carga máxima (1RM). A reprodutibilidade dessa técnica tem demonstrado valores de correlação teste-restes superiores a $0,9^{15}$.

A velocidade foi avaliada segundo os protocolos descritos na literatura específica $^{16,17}$, realizando a tomada de tempo de deslocamento contínuo em linha reta na distância 30 metros, em um campo de futebol de grama natural. As tomadas de tempo foram realizadas por fotocélulas. Foi usada a distância de $30 \mathrm{~m}$ para avaliar a capacidade de velocidade máxima linear do atleta.

Para análise estatística dos dados, foi adotada a estatística descritiva, com as médias, desvio padrão e intervalo de confiança. Para verificarmos a normalidade da distribuição foi utilizado o teste de Shapiro-Wilk (SW). O valor $\mathrm{p}$ $\leq 0,05$ foi adotado para estabelecer a significância estatística para todas as comparações. Como a normalidade não foi atendida (velocidade), foi utilizado o teste não paramétrico de correlação de Spearman para analisar as relações entre as variáveis investigadas. Todos os cálculos estatísticos foram realizados utilizando o software SPSS 22.0 (IBM, Inc). A chance da correlação ser considerada positiva, nula ou negativa foi calculada e avaliada qualitativamente da seguinte forma: < $1 \%$ trivial (quase certamente não); 1-5\% muito improvável; 5-25\% improvável; 25-75\% possível; 7595\% provável; 95-99\% muito provável e > $99 \%$ quase certamente sim. Se os valores negativos e positivos apresentassem resultados $>5 \%$, a inferência era considerada inconclusiva ${ }^{18,19,20}$. Essa análise foi realizada por meio das planilhas disponíveis em: http://sportsci.org/2006/wghcontrial.htm 27 . 


\section{RESULTADOS E DISCUSSÃO}

Os dados não apresentaram distribuição normal $(\mathrm{p}<0,05)$ para velocidade.

Tabela 1. Análise descritiva expressa em média, desvio padrão (DP) e intervalo de confiança (IC95\%) da força máxima (1RM) e velocidade máxima linear (Vel30)

\begin{tabular}{lccc}
\hline & & \multicolumn{2}{c}{$95 \mathbf{I C \%}$} \\
\hline & Média \pm DP & Inferior & Superior \\
\hline $\mathbf{1 R M}$ & $99 \pm 2,09$ & 94,59 & 103,41 \\
Vel30 & $4,20 \pm 0,03$ & 4,13 & 4,28 \\
\hline
\end{tabular}

A tabela 1, apresenta média, desvio padrão e intervalo de confiança das variáveis analisadas. A tabela 2, apresenta a inferência qualitativa e os valores da correlação entre as duas variáveis, assim como sua significância.

Tabela 2. Valores de inferência qualitativa, correlação de Spearman e significância das variáveis força (1RM) e velocidade (Vel30)

\begin{tabular}{lc}
\hline & 1RM vs Vel30 \\
\hline Inferência qualitativa & Provável \\
Correlação Spearman & $r=-0,367$ \\
Significância & $p=0,134$ \\
\hline$r$ : valor da correlação; $p$ : valor da significância
\end{tabular}

A figura 1, mostra as correlações entre velocidade máxima linear (Vel30) e força máxima (1RM) $(r=-0,37 ; \mathrm{p}>$ $0,05)$.

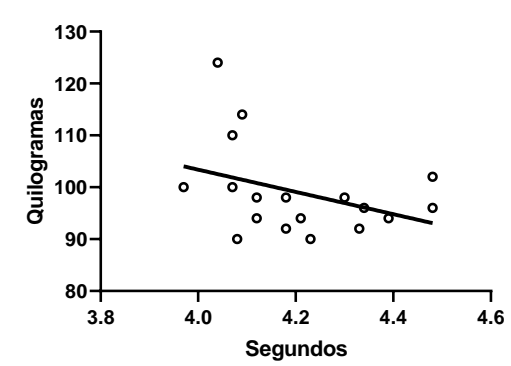

Figura 1 - Relação entre teste de força máxima medida através do teste de repetição máxima (1RM expresso em quilogramas) e velocidade máxima linear medida pelo Sprint de 30 metros (Vel30 expresso em segundos).

\section{CONCLUSÃO}

Não foi verificada forte correlação entre o teste de força máxima, analisado pelo 1RM e a velocidade máxima linear, analisada pelo teste de Sprint de $30 \mathrm{~m}$ em jovens futebolistas.

\section{REFERÊNCIAS}

1. Stolen T, Chamari K, Castagna C, Wisloff U. Physiology of soccer: an update. Sports Med. 2005;35(6):501-36. 2. Mohr M, Draganidis D, Chatzinikolaou A, Barbero-Álvarez JC, Castagna C, Douroudos I, et al. Muscle damage, inflammatory, immune and performance responses to three soccer games in 1 week in competitive male players. Eur J Appl Physiol. 2016;116(1):179-93.

3. ACSM's guidelines for exercise testing and prescription / American College of Sports Medicine ; senior editor, Linda S. Pescatello ; associate editors, Ross Arena, Deborah Riebe, Paul D. Thompson. 9th ed. 2013.

4. National Strength And Conditioning Association. Youth resistance training: Updated position statement paper from the National Strength and Conditioning Association. Journal of Strength and Conditioning Research. 2009; 23: S60S79. 
5. Seynnes OR, De Boer M, Narici MV. Early skeletal muscle hypertrophy and architectural changes in response to highintensity resistance training. $\mathrm{J}$ Appl Physiol 2007; 102:368-373.

6. Tillin N, Folland J. Maximal and explosive strength training elicit distinct neuromuscular adaptations, specific to the training stimulus. Eur. J. Appl. Physiol. 2014; 114, 365-374.

7. Folland, JP, Williams AG. The adaptations to strength training. Sport Med. 2007; 37, 145-168.

8. Buckner SL, Jessee MB, Mattocks KT, Mouser JG, Counts BR, Dankel SJ, Loenneke JP. Determining strength: a case for multiple methods of measurement. Sport Med. 2017; 47, 193195.

9. Baechle TR, Earle RW. Essentials of strength training and conditioning, Champaign: Human Kinetics, 2000.

10. Picerno P, Iannetta D, Comotto S, Donati M, Pecoraro F, Zok M, Tollis G, Figura M, Varalda C, Di Muzio D, Patrizio F, Piacentini MF. 1RM prediction: a novel methodology based on the force- velocity and load-velocity relationships. Eur. J. Appl. Physiol. 2016. 116, 2035-2043.

11. Muñoz-López M, Marchante D, Cano-Ruiz MA, Chicharro JL, Balsalobre-Fernández C. Load, force and power-velocity relationships in the prone pull-up exercise. Int. J. Sports Physiol. Perform. 2017. 2, 1-22.

12. Cormie P, McGuigan MR, Newton RU. Developing maximal neuromuscular power: part 2-training considerations for improving maximal power production. Sports Med. 2011; 41: 125-146.

13. Weyand, PG, Sternlight, DB, Bellizzi, MJ, and Wright, S. Faster top running speeds are achieved with greater ground forces not more rapid leg movements. J Appl Physiol 2000. 89: 1991-1999.

14. World Medical Association. World Medical Association Declaration of Helsinki: Ethical principles for medical research involving human subjects. JAMA. 2013;310(20):2191-4.
15. Ritti-Dias RM, Avelar A, Menêses AL, Salvador EP, Pereira DA Silva DR, Cyrino ES. Segurança, reprodutibilidade, fatores intervenientes e aplicabilidade de testes de 1-RM. Motriz, 2013;19(1):23142.

16. Little T, Williams AG. Specificity of acceleration, maximum velocity, and agilityin professional soccer players. J Strength Cond Res. 2005; 19(1): 76-8.23. 17. Young WB, McDowell MH, Scarlett BJ. Specificity of Sprint and Agility Training Methods. Journal of Strength and Conditioning Research, 2001; 15(3), 315-319.

18. Batterham AM, Hopkins WG. Making meaningful inferences about magnitudes. Sportscience. 2005;9:6-13. Disponível

em: http://sportsci.org/jour/05/ambwgh.htm 19. Hopkins WG. A spreadsheet for analysis of straightforward controlled trials. Sportscience. 2003. [acesso em 20 set de 2019]. Disponível em: http://sportsci.org/resource/stats/generali ze.html\#calculate

20. Hopkins W, Marshall S, Batterham A, Hanin J. Progressive statistics for studies in sports medicine and exercise science. Med Sci Sports Exerc. 2009;41(1):3-13.

21. Hunter, JP, Marshall, RN, and McNair, PJ. Relationships between ground reaction force impulse and kinematics of sprint-running acceleration. J Appl Biomech. 2005. 21: 31-43.

22. McBride JM, Blow D, Kirby TJ, Haines TL, Dayne AM, Triplett NT. Relationship between maximal squat strength and five, ten, and forty yard sprint times. J Strength Cond Res. 2009 Sep;23(6):1633-6.

23. Comfort P, Stewart A, Bloom L, Clarkson B. Relationships between strength, sprint, and jump performance in well-trained youth soccer players. J Strength Cond Res. 2014 Jan;28(1):173-7 24. Comfort, P, Bullock, N, Pearson, SJ. A comparison of maximal squat strength and 5-, 10-, and 20-meter sprint times, in 
athletes and recreationally trained men. J Strength Cond Res. 2012; 26: 937-940. 25. Wisloff U, Castagna C, Helgerud J, Jones R, Hoff J. Strong correlation of maximal squat strength with sprint performance and vertical jump height in elite soccer players. Br J Sports Med. 2004; 38: 285- 288.

26. Abad C, Cuniyochi R, Kobal R, Gil S, Pascoto K, Nakamuraa F, Loturco I. Efeito do destreinamento na composição corporal e nas capacidades de salto vertical e velocidade de jovens jogadores da elite do futebol brasileiro. Rev Andal Med Deporte. 2016;9(3):124-130.

Informações do artigo / Information of this article:

Recebido: 10/11/2019

Aprovado: 14/11/2019

Publicado: 08/04/2020

Received: 10/11/2019

Approved: 14/11/2019

Published: 08/04/2020

Ezequias Pereira Neto

ORCID: 0000-0003-3210-0754.

Como citar esse artigo / How to cite this article:

Pereira Neto, E. et al. Correlação entre medidas de força e velocidade em jovens futebolistas. Arq. Bras. Ed. Fís., Tocantinópolis, v. 2, n. 2, Ago./Dez., p. $36-42,2019$. 